\title{
Investigation on Coupling Reaction of Fragments Towards Total Synthesis of Pyrenophorin
}

\author{
Jaqueline Rosa Barbosa (IC), Maurício Moraes Victor (PQ)*.
}

Organic Chemistry Department, Chemistry Institute, Federal University of Bahia (UFBA), CEP: 40170-290

*mmvictor@ufba.br

Keywords: Pyrenophorin, Yamaguchi coupling, Organic Synthesis.

\section{INTRODUCTION}

Molecules belonging to the medium and large size rings lactones have attracted considerable attention from synthetic chemists due to their interesting biological properties. Due these structural features and importance, we have accepted the challenging to prepare Pyrenophorin 1 (Figure 1), a symmetric sixteen-membered diolide isolated from Pyrenophora avenae ${ }^{1}$ which displays potent antifungal activity. In our synthetic strategy to obtain a half-part of this molecule, we found several problems to perform a coupling reaction of fragments 3 and 4 . Several efforts were focused on coupling reaction to construct a fragment 2 . This work shows our results in this direction.

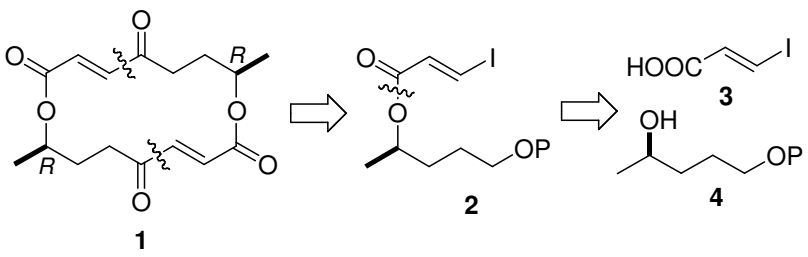

Figure 1. Retrosynthetic analysis.

\section{RESULTS AND DISCUSSION}

Our efforts began with the choice of best silylprotecting group of (+/-)-1,4-pentanediol (5). Due chemoselective requirement, TBS, TIPS, and TBDPS were tested as primary hydroxy protecting groups. The best results were reached at lower temperature reaction $\left(0^{\circ} \mathrm{C}\right)$ performed with TIPSCI as silylating agent (Scheme 1).<smiles>CC(O)CCCO</smiles>
5

$$
\frac{\mathrm{RCl}, \mathrm{Et}_{3} \mathrm{~N}}{\text { DMAP, } \mathrm{CH}_{2} \mathrm{Cl}_{2}}
$$

$\mathrm{OH}$

4a (R=TBS) $43 \%$
4b (R=TIPS) $85 \%$

4c (R=TBDPS) $69 \%$

Scheme 1. Selective silylation of 5 .

After preparation of 1-O-protected diols, we decided to investigate a suitable methodology to coupling the monosilylated alcohols $4 a-c$ and E-iodo acrylic acid (3). ${ }^{2}$ As our first strategy to employ carbodiimides has failed (yields $<10 \%$ ), we focused our attention on Yamaguchi methodology (Scheme 2):

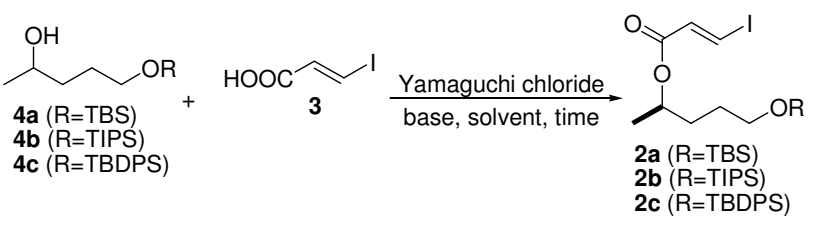

Scheme 2. Yamaguchi coupling.

We tested 2 different methodologies with Yamaguchi chloride: a classical procedure, ${ }^{3}$ where a mixed anhydride was isolated before addition of alcohol; and a one-pot procedure, without isolation of intermediate. ${ }^{4}$ Various modification were performed, as use of different bases $\left(\mathrm{Et}_{3} \mathrm{~N}\right.$ and DIPEA), solvents (DCM, toluene, THF), temperatures (from $0{ }^{\circ} \mathrm{C}$ to 65 ${ }^{\circ} \mathrm{C}$ ), and reaction times (until 4 days). The best results obtained were a classical procedure at room temperature, with $\mathrm{Et}_{3} \mathrm{~N}$ and alcohol 4a (15h, 34\% yield of $\mathbf{2 a}$ ), and a one-pot Yamaguchi reaction at 0 ${ }^{\circ} \mathrm{C}$, with DIPEA and alcohol $\mathbf{4 b}$ in toluene (3d, $48 \%$ yield of $\mathbf{2 b}$ ). Higher temperatures $\left(65^{\circ} \mathrm{C}\right)$ in one-pot reaction lead to higher yield isolated (81\%), but unfortunately on impure form. Some attempts to prepare a acyl chloride from acid 3 were also investigated, but ester was not isolated.

\section{CONCLUSION}

We investigated the coupling reaction between $E$ iodo-acrylic acid (3) and O-sylil-protected 1,4pentanediols $\mathbf{4 a - c}$ on various conditions. The best result obtained was $48 \%$ yield with one-pot Yamaguchi reaction.

\section{ACKNOWLEDGEMENTS}

GPSQ for chemical support, and prof. Luiz C. Dias for precious collaboration.

\section{REFERENCES}

${ }_{1}^{1}$ Ishibashi, K.; Agric. Chem. Soc. 1961, 35, 257.

2 Cox, L. R.; DeBoos, G. A.; Fullbrock, J. J.; Percy, J. M.; Spencer, N.; Tetrahedron: Asymmetry 2005, 16, 347.

${ }^{3}$ Inanaga, J.; Hirata, K.; Saeki, H.; Katsuki, T.; Yamaguchi, M.; Bull. Chem. Soc. Jpn. 1979, 52, 1989.

${ }^{4}$ Navarro, I.; Basset, J.-F.; Hebbe, S.; Major, S. M.; Werner, T.; Howsham, C.; Brackow, J.; Barrett, A. G. M.; J. Am. Cgem. Soc. 2008, 130, 10293. 